\title{
La mejor alternativa económica para el puerto de Barranquilla. Un Reto Empresarial
}

\section{The best economic alternative for the port of Barranquilla. A Business Challenge}

\author{
DOI: http://dx.doi.org/10.17999/bilo
}

Artículo de Investigación Científica. Fecha de Recepción: 29/05/2020. Fecha de Aceptación: 25/06/2020

\author{
Shirley Llinás-Rojas \\ Universidad de la Costa CUC. Barranquilla, (Colombia) \\ sllinas@cuc.edu.co
}

Para citar este artículo:

S. Llinás Rojas "La mejor alternativa económica para el puerto de Barranquilla. Un Reto Empresarial ”, BILO, vol. 2, no. 1, 2020. DOI: http://dx.doi.org/10.17999/bilo

\section{Resumen}

En la actualidad las compañías buscan la optimización de sus recursos para lograr ser más rentable y lograr la eficiencia en sus procesos. A la hora de lanzar un proyecto se debe tener en cuenta el micro y macro entorno de la ciudad y país.

En el caso del puerto de barranquilla se debe aprovechar su posición geográfica ya que esto nos permite tener contacto con todas las regiones del país y el exterior; para esto se deben estudiar diferentes alternativas de acuerdo a la situación actual del puerto, para lograr que este sea más eficiente y competitivo frente a los puertos nacionales e internacionales.

Precisamente con este estudio se busca plantear una solución para mejorar el puerto de Barranquilla, con el fin de lograr mayor competitividad frente a otras zonas portuarias y así poder generar mayores ingresos aprovechando su posición geográfica dada por río y mar.

Dentro de esta investigación se tomó como objeto de estudio el Puerto de Barranquilla junto con los datos dado por un estudio de caso (obtenido de la Universidad de la Costa) referente al mismo y se hizo un análisis de datos probabilístico con índices de bondad financiera (VPN), generándose como decisión la alternativa de reducción de necesidades de dragado con obras hidráulicas ya que generan el menor costo para realizar la obra.

La mayor parte del problema viene dado por la sedimentación que trae el río que no permite el flujo continuo de embarcaciones y para eso se busca realizar obras en boca de ceniza e isla 1972 para así poder generar solución a la desventaja y generar mayores ingresos y fuentes de empleo.

\section{Palabras clave}

Puerto; Barranquilla; dragado; costos; inversión; toneladas; carga; alternativa.

\footnotetext{
Abstract

Currently, companies are looking for the optimization of their resources to be more profitable and achieve efficiency in their processes. When a project is accepted, the micro and macro environment of the city and country must be taken into account.

In the case of the port of Barranquilla, its geographical position must be used since this allows us to have contact with all regions of the country and other countries; for this we must study different alternatives according to the current situation of the port, to ensure that this would be more efficient and competitive against national and international ports.
} 
The idea with this study is to propose a solution to improve the port of Barranquilla, in order to be a more competitive compared to other port areas and thus be able to generate greater income taking advantage of its geographical position given by river and sea. In this investigation, the Port of Barranquilla was taken as an object of study along with the data given by the case study concerning it and a probabilistic data analysis with financial goodness indices was made (VPN). The decision was the alternative to reduce dredging needs with hydraulic works were taken as they generate the lowest cost to carry out the work.

Most of the problem is due to the sedimentation that the river brings that does not allow the continuous flow of vessels and for that purpose it is sought to carry out works in the mouth of ash and island 1972 in order to generate a solution to the disadvantage and generate greater income and sources of employment.

\section{Key Words}

Port; Barranquilla; dredged; costs; investment; tons; load; alternative.

\section{INTRODUCCIÓN}

Ser competitivo y eficiente dentro de las sociedades económicas es el principal reto que tiene toda organización para obtener reconocimiento y mayores utilidades. En nuestro caso, concerniente al puerto de Barranquilla, el constante movimiento de intercambio comercial ha llevado a los puertos a que hagan cambios estructurales que le permitan ser competitivos frente a otros puertos y sobre todo por la globalización que se ha venido dando, así como los tratados de libre comercio que han permitido una mayor relación económica entre países y en la que el transporte marítimo y fluvial ha tenido mayor auge por la facilidad de transportar las mercancías y por reducción de costos.

Además, la tecnología ha dado un gran paso de innovación y avance que permitiría de manera más fluida investigar sobre el comportamiento económico de los puertos en el mundo, así como implementación de máquinas que permitan ayudar al trabajo duro como es el caso del dragado, buscando soluciones a alternativas ya existentes o generando nuevos retos en el transporte por agua.

El transporte marítimo y fluvial es importante ya que la mayoría de operaciones de intercambio de mercancías se realizan por este medio y eso hace que haya prioridad para los puertos, por lo que se debería trabajar de manera constante en la mejora de las infraestructuras de los mismos para que haya equilibrio entre importadores y exportadores tanto nacionales como internacionales. El puerto de Barranquilla posee una estructura geográfica privilegiada ya que en comparación con los demás puertos del país tiene conexión con el Río Magdalena y con el Mar Caribe; lo que lo convierte en un punto estratégico para el transporte de mercancías dentro y fuera del país. De acuerdo a estas ventajas geográficas junto con otros factores (económicos, sociales, tecnológicos, entre otros), se analizarán alternativas de mejoras ya que se presentan problemas de calado en el río, a la que se debe buscar solución para que se pueda lograr adquirir un puerto eficiente y competitivo; generando mayores ingresos a la ciudad junto a más empleos y siendo reconocidos a nivel global.

\section{REVISIÓN LITERARIA}

La infraestructura de los puertos es quizá una de las principales problemáticas que se presenta ya que cada vez hay un auge en la tecnología que nos deja un paso atrás a seguir creciendo, innovando y mejorando continuamente los procesos que se llevan dentro de él.

Es necesario contar con infraestructuras adecuadas para los requerimientos de los procesos de internacionalización que contribuyen en el aprovechamiento de oportunidades del entorno. [1]

Aunque de manera general, se puede observar que si se quiere ser competitivo fuertemente en la industria portuaria y naviera hay que ajustarse a esa innovación clave que se viene mencionando ya que el crecimiento de la economía y la tecnología ha traído junto con ellas modificaciones y/o mejoras en los barcos los cuales suelen ser cada vez más grandes por lo tanto los puertos deberían encontrarse en la capacidad de recibir dichos barcos pero no hay muelles que se ajusten a esas condiciones y además el calado se presenta como un segundo problema. Esto nos deja ver que en la organización portuaria y fluvial, se necesita de manera urgente crear esquemas de mejoras y crecimiento continuo para lograr objetivos de competencias fuertes.

La globalización mundial genera que cada día se vayan generando soluciones a los problemas que se presentan en la sociedad principalmente en lo que genera mayor economía y competitividad, como es el caso de Barranquilla, su puerto.

La Sociedad Portuaria Regional de Barranquilla, Puerto Marítimo y Fluvial fue creada el 22 de diciembre de 1936, con fin principal de fomentar las actividades comerciales por vía fluvial, aérea y terrestre el interior del país. Inicialmente fue administrado por la Sociedad de Mejoras Públicas de la ciudad, posteriormente la administración queda a cargo de la empresa Puertos de Colombia. [1] 
Estas actividades se ven cesadas ya que no hay flujo de grandes embarcaciones a comparación de otros puertos por el problema de calado. Esto es una gran desventaja ya que se ve disminuida la entrada y salida comercial que genere mayores ingresos y mayores empleos. Para esto se plantean proyectos para la recuperación del Río Magdalena que permita tener una mayor conexión con el Mar Caribe para que se pueda crear un vínculo comercial con el interior del país.Esta recuperación del río es algo importante ya que él cuenta con una cuenca hidrográfica de $262.000 \mathrm{~km} 2$ donde hay transición de 18 departamentos de 32 que tiene el país. Este genera aproximadamente $85 \%$ del PIB nacional. Lo anterior lo convierte en la principal arteria fluvial de Colombia, pese a no ser ni el río más largo ni el más caudaloso [2]. De los más de 1.500 $\mathrm{km}$ que recorre el río, éste es navegable en aproximadamente $800 \mathrm{~km} \mathrm{(52 \% ),} \mathrm{presentando} \mathrm{los} \mathrm{mayores} \mathrm{problemas} \mathrm{de}$ navegabilidad entre Puerto Berrío (Santander) y La Dorada (Caldas) [2].

El río Magdalena presenta inconvenientes de acumulación de sedimentos y caudal fuerte. Su caudal se encuentra entre $1.500 \mathrm{~m}^{3} / \mathrm{s}$ y $11.000 \mathrm{~m}^{3} / \mathrm{s}$.

El transporte total de sedimentos llega a 200 millones de toneladas por año. Es decir, el Magdalena arrastra, en promedio, $0,9 \mathrm{~kg}$ de sedimentos por cada metro cúbico de agua, lo cual lo ubica como el octavo del mundo en cantidad de residuos transportados. [2]. Cuando presenta esta problemática, el Puerto de Barranquilla conectado directamente con este río, es el cuarto puerto más importante ya que permite la expansión y la comunicación con el interior y exterior del país. El comercio marítimo se encuentra repartido entre Barranquilla, Santa Marta y Cartagena, siendo las dos últimas de mayor competitividad por la profundidad de sus puertos. Barranquilla con su puerto tiene problemas de calado que se encuentra en máximo 28 pies, dado a la acumulación de los sedimentos que son arrastrados cada año y que son productos de la deforestación y de la erosión que se da en la cordillera de los Andes. Esta sedimentación es la que reduce la profundidad del río reduciendo la entrada de barcos con calados grandes, transportándose una misma carga en una mayor cantidad de barcos de menor calado, generando así mayor gastos y disminuyendo la ventaja de interconexión que se tiene.

Toda esta problemática de la sedimentación la lleva Cormagdalena, quien se encarga del mantenimiento del canal, pero no hay quien se encargue directamente del dragado que es el punto a mejora ya que, si se aumenta el calado permisible, va a generar aumento en la capacidad del puerto. En general, se espera que el puerto de Barranquilla crezca económicamente en los próximos años gracias a los proyectos de recuperación de navegabilidad que existen para mejorar la efectividad de prestación de servicios en comercio nacional e internacional de mercancías por vía marítima y fluvial.

\section{- Indicadores de tasa de rendimiento}

La tasa social de descuento mide el costo al cual una sociedad está dispuesta a sacrificar el consumo presente por el consumo del mañana. Entre más alta sea la tasa de descuento, mayor disposición de la sociedad para consumir más en el presente que en le mañana. [3], [4], [5].

Por medio de la página del Departamento Nacional de Planeación se obtuvo la tabla de descuentos recomendada, tomándose dicho descuento o TSD como el valor presente neto (VPN) de este estudio (Valor para Colombia) :

\begin{tabular}{|c|c|c|c|c|}
\hline Puis & Metodalogill & Referencil & TSD Anterior & $\begin{array}{c}\text { TSD } \\
\text { Recome ndadu } \\
\text { Artuulmente }\end{array}$ \\
\hline \multicolumn{5}{|c|}{ Latinoamerica } \\
\hline Costa Rica & - & Cartes, Contreras y cruz (2010) & $12 \%$ & $12 \%$ \\
\hline Mexico & Harberger (1972) & Campos, Serebrisky y Suarez-Alemin (2015) & $12 \%$ & $10 \%$ \\
\hline Perú & pp & Campos, Serebrisky y Suarez-Alemin (2015) & $10 \%$ & $9 \%$ \\
\hline Colombia & Harberger (1969) & DNP & $12 \%$ & $9 \%$ \\
\hline Chile & pp & Campos, Serebrisky y Suarez-Alemin (2015) & $10 \%$ & $6 \%$ \\
\hline
\end{tabular}

Tabla 1. Tasas de descuento para proyectos de inversión en Latinoamérica

\section{- Tarifas del puerto}

Como toda sociedad financiera, el puerto de Barranquilla maneja unos valores o tarifas con los cuales generan sus ingresos ya sea por carga o por contenedores. 
A continuación, se muestra la tabla de tarifas para los contenedores que será usada más adelante en el estudio

\begin{tabular}{c|c} 
Contenedores: & \\
\hline Contenedor lleno 20' Normal & 95,00 \\
\hline Contenedor Ileno 40' Normal & 125,00 \\
\hline Contenedor vacio 20' & 25,00 \\
\hline Contenedor vacio 40 & 25,00 \\
\hline
\end{tabular}

Tabla2. Tarifas uso de instalaciones a la carga (contenedores)

\section{METODOLOGÍA}

En este trabajo se hizo una investigación de tipo cuantitativo y cualitativo para poder hacer el análisis numérico que determinan los resultados principales de la investigación. Los datos aquí estudiados fueron interpretados utilizando la herramienta de EXCEL y fueron recolectados por intermedio de explicación directa de los mismos por medio de un PDF facilitados por una docente de la cátedra de ingeniería económica de la Universidad de la Costa.

El valor de la Trema o VPN fue obtenida de la página de Departamento Nacional de Planeación (DNP) que fue del 9\% utilizada para el indicador de bondad financiera correspondiente al TSD de Colombia, así como el valor por contenedor usado para determinar el gasto de mantenerlos en el puerto que fue de US95 para un contenedor lleno de 20.

Se tomaron como alternativas para el estudio las siguientes opciones:

- Alternativa 1: Reducción de las necesidades de dragado con obras hidráulicas.

- Alternativa 2: Construcción del puerto de aguas profundas de Bocas de Ceniza.

- Alternativa 3: Puente Pumarejo más alternativa 1 y 2.

Como fuente adicional se tomó información de páginas web, tesis, documentos relacionados con estudios de puertos y artículos relacionados con la información del Puerto de Barranquilla.

\section{RESULTADOS}

Como primer resultado del estudio, se obtuvo una proyección de datos en Excel de las toneladas por años donde se observa una tendencia creciente en la carga de escenario en miles de toneladas por año, durante los siguientes 5 años (año16- año 20). Se puede ver que los siguientes 5 años son de crecimiento para la cantidad de tonelada que se transportaría dentro de los puertos por lo que es necesario buscar alternativas de mejoras o estudiar las alternativas propuestas para poder buscar una solución óptima que va de acuerdo a las necesidades de control de gastos o a cada condición que presenten los datos numéricos del caso.

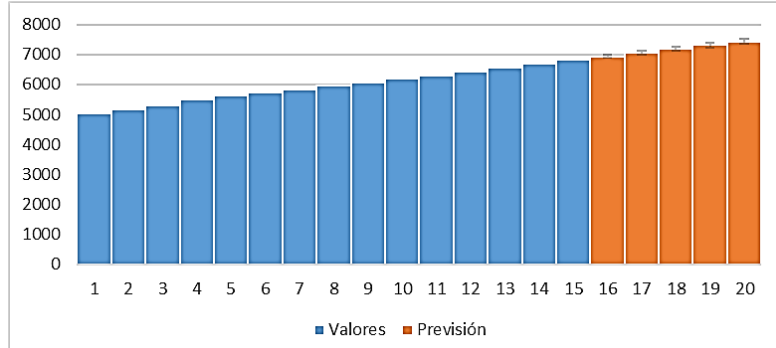

Figura 1. Proyección de toneladas a transportar en el puerto 


\begin{tabular}{|r|r|r|}
\hline Escala de tiempo & Valores & Previsión \\
\hline 1 & 5023 \\
\hline 2 & 5165,62 \\
\hline 3 & 5311,76 \\
\hline 4 & 5511,5 \\
\hline 5 & 5616,18 \\
\hline 6 & 5723,43 & \\
\hline 7 & 5833,3 \\
\hline 8 & 5945,87 & \\
\hline 9 & 6061,2 \\
\hline 10 & 6179,35 & \\
\hline 11 & 6300,4 \\
\hline 12 & 6424,41 & \\
\hline 13 & 6551,46 \\
\hline 14 & 6681,63 & \\
\hline 15 & 6814,99 & \\
\hline 16 & & 6937,90859 \\
\hline 17 & & 7061,83705 \\
\hline 18 & & 7185,7655 \\
\hline 19 & & 7309,69396 \\
\hline 20 & & 7433,62241 \\
\hline
\end{tabular}

Tabla 3. Proyecciones numéricas de toneladas a transportar en el puerto

Continuando con el estudio de caso, se tienen 3 alternativas las cuales arrojaron cada una un resultado definitivo que permitirá tomar la decisión más adecuada.

En el caso de la Alternativa 1 se manejaron dos escenarios de construcción para llevar a cabo. El primer escenario que sería realizar obras en Isla 1972 y Bocas de Ceniza con espolones de acero y núcleo en Bolsacretos, cierre de dique y espolón en rocas con un Costo Total de US 18 742.224 y para la opción 2 o segundo escenario que consiste en construir todas las obras en roca con un Costo Total de US 27 160.693. Ambas opciones dan una profundización de 36 pies (aumento de 8 pies).

De manera simple y por opción de ahorro se tomaría la opción 1 porque genera menor costo porque ambas igual van a profundizar el canal a 36 pies que es lo que se requiere para disminuir el problema de calado en el río.

Luego, analizando con el VPN tomando una TREMA del 9\%, valor dado por DNP para el estudio de proyectos, con ayuda de la herramienta Excel y con los datos recolectados por información suministrada de los costos recurrentes de mantenimiento para las dos opciones de construcción de la Alternativa 1, se obtuvo que para la opción 1 se genera un VPN negativo de US 334.962,00 y para la opción 2 se genera un VPN negativo de US 823.650,00

Se toma el VPN que genera menor costo que es el de la opción 1.

Luego, teniendo la probabilidad del $65 \%$ de que la alternativa 1 logre sus objetivos frente a la del $35 \%$ que no (datos suministrados), genera un valor de US $\mathbf{3 0 0 . 0 0 0}$ para suplir la demanda de contenedores. Esto determinado luego que se tuviera que al llevarse a cabo la Alternativa iba a generar un beneficio neto de US 1.000 .000 en el año que se cierre el muelle y en el caso contrario, anualmente deberían invertir en el dragado anual por un costo de US 1.000.000. Además, se tiene que la probabilidad que se cumpla la demanda dado que se lleve la Alternativa 1 es de $40 \%$, por lo que realizando un árbol de decisión con las anteriores probabilidades se obtuvo el costo dado para suplir la demanda de contenedores.

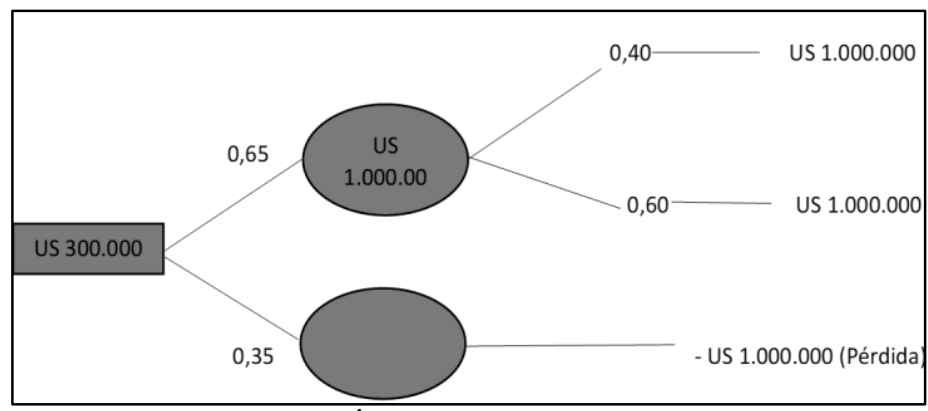

Figura 2. Árbol de decisión beneficios

La Alternativa 2, se encuentra ramificada en dos fases. La primera (Fase 1) que consiste en construir un terminal exclusivo para transportar carbón ya que actualmente no pueden entrar al puerto por restricciones de calado que hay en el canal de acceso y el segundo (Fase 2) que consiste en realizar inversiones en infraestructura para movilizar contenedores y otros productos además de carbón. 
Para la fase 1 se tenía una inversión inicial de US 180.000 .000 con costos anuales del 5\% de la inversión inicial (US 9.000.000) para unas barcazas donde están incluidas las operaciones de dragados, estructuras marinas, equipo portuarios y una inversión inicial de US 5.000.000 con costos anuales del 4\% de la inversión inicial (US 200.000) para un terminal de transbordo para camiones para transferir la carga a las barcazas. A partir de estos datos se halló el VPN con Excel, se obtuvo que para la construcción de las barcazas se genera un VPN negativo de US 252.546.195,87 y para el terminal de transbordo para camiones se genera un VPN negativo de US 6.612.137,69. Deduciéndose que sale más económico construir el terminal de transbordo que construir las barcazas, sabiendo que ambas se encuentran interconectadas.

Además, conociendo que la probabilidad que se dé el proyecto de la barcaza es del 30\% mientras que se siga con los camiones es de 70\%, y conociendo los valores de VPN calculados, se multiplican las respectivas probabilidades con dichos VPN y se obtiene un VPN general de US 25.969.105,1. Lo que nos indica un alto costo al realizar esta fase 1 de la Alternativa 2.

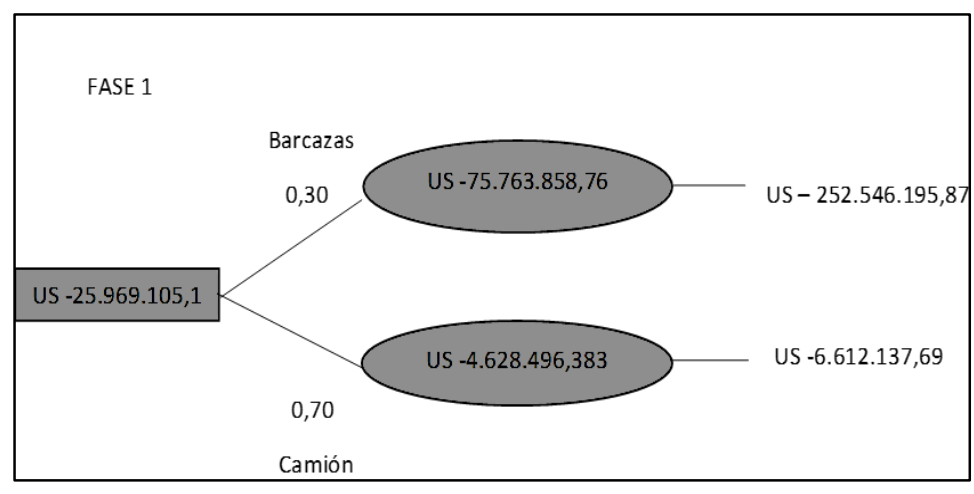

Figura 3. Árbol de decisión Valor Presente Neto Alternativa 2 Fase 1

Para la fase 2 se tiene una inversión de US 300.000.000 con costos anuales del 6\% sobre la inversión inicial (US 24.000.000) obteniéndose de este un VPN negativo de US 211.180.747,46.

Se conoce que la probabilidad de que el proyecto se de en Bocas de Ceniza es del $40 \%$ mientras que se de en Cartagena es del $60 \%$, pero además se tiene que la probabilidad de que Cartagena reciba un $80 \%$ de los contenedores es del $20 \%$ frente a que reciba Bocas de ceniza un $60 \%$ de los contenedores es del $40 \%$. De acuerdo a la información de la Superintendencia de Puertos que la posible sobreoferta de contenedores será de 7,2 millones al año para ambos puertos (Cartagena recibiría 5.760.000 contenedores aproximadamente y Bocas de Ceniza 4.320.000 contenedores) y por medio de un árbol de decisión donde el costo por contenedor de 20 pies de carga dentro del puerto es de US 95 (valor tabla obtenida de la página de la sociedad portuaria), se obtuvo que el costo sería de US 262.656,00 (millones de dólares).

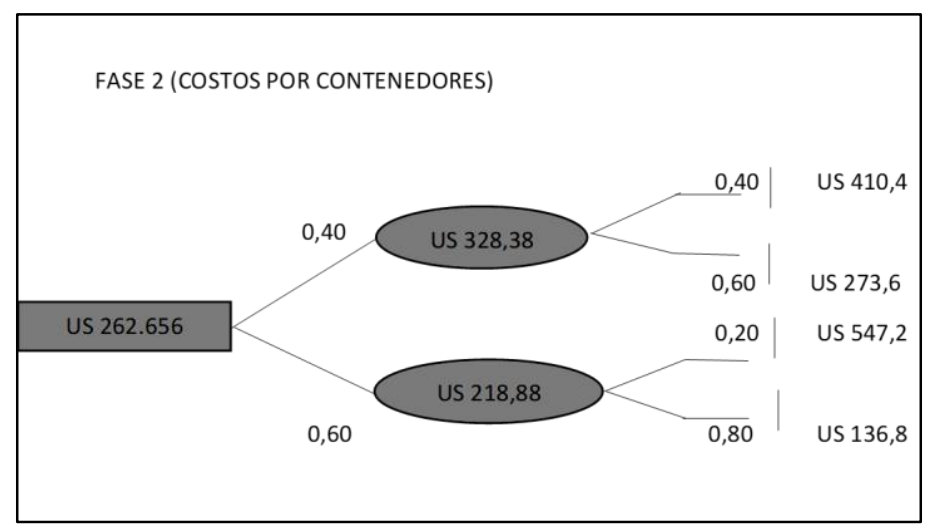

Figura 4. Árbol de decisión Costo por contenedores Alternativa 2 Fase 2

Para la Alternativa 3, que consiste en llevar a cabo la alternativa 1 y 2 al mismo tiempo con la construcción del puente con dos condiciones adicionales. 
La primera consiste en hacer un puente colgante el cual realizarlo costaría US 60.000 .000 y la segunda consiste en un túnel o paso subfluvial que tendría un costo de US 70.000.000. Si tocara tomar una decisión de tipo monetario se tomaría la opción 1 por ser más económica, pero en este caso también se debe tener en cuenta otros factores como cantidad de barcos que pasarían por el río y su capacidad; además hay otros proyectos que pueden generar mayor rentabilidad e incluso se podría hacer otro tipo de inversión ya que actualmente se encuentra en proceso la fase final del nuevo puente Pumarejo, por lo que se debería pensar en solucionar el problema de calado para que los buques puedan navegar por el río sin dificultad haciendo uso del trayecto del río donde está construido el nuevo puente para que haya mayor conexión entre puertos y ciudades.

De esa manera, teniendo de base el funcionamiento del nuevo puente que ya genera un cambio para la ciudad de Barranquilla, se analizan las dos primeras Alternativas, realizándose en este caso por costos reducidos. Es decir, hacer el análisis de costos por medio del cual se escoge la opción que genere menos gastos y que a su vez maximice la competitividad marítima y fluvial que traería consigo mayores ingresos a la ciudad y generación de oportunidades.

En el caso de la alternativa 1 se escogería la opción 1 que es la de profundizar y hacer pilotes de acero y núcleo en Bolsacretos, dique de cierre en arena y espolón en rocas ya que generó el menor costo al hacer uso del indicador de bondad financiera Valor Presente Neto (VPN).

En el caso de la alternativa 2 se escogería la fase 1 en la construcción del terminal exclusivo para el carbón ya que también generó el menor costo al hacer uso del indicador de bondad financiera VPN pero analizando otros factores que no solo sean los económicos podríamos escoger la fase 2 ya que genera mayor apertura de mercado porque permitiría mayor intercambio de carbón pero a su vez de otros productos en fin de convertir al puerto en un Hub o troncoalimentador compitiendo así con la Sociedad Portuaria Regional de Cartagena quien es la que cumple esta función para la mayor parte del mercado caribeño. A su vez, esto llegaría generar mayores ingresos aunque el costo de la inversión sea alta. Comparando ahora entre la Alternativa 1 (opción 1) y Alternativa 2 (fase 2), tomaría la alternativa 1, basados en datos numéricos y cálculos realizados con el indicador de bondad financiera VPN, ya que si se lleva a cabo va a generar ganancias anuales de US 1.000.000 siempre que se lleve a cabo, obteniéndose rentabilidad por US 300.000. Es decir, sería factible tomar esta alternativa porque al llevar las obras e la isla 1972 y luego en Bocas de Ceniza se minimizará el dragado ya que en ellas se generaría un efecto de auto lavado que arrastra el sedimento a su vez aumentando el calado de 28 a 36 pies, permitiendo el paso de las embarcaciones de manera más fluida, aprovechándose a su vez la construcción del nuevo puente Pumarejo que va a permitir el paso de esas embarcaciones para el transporte de mercancías.

No obstante, y como se mencionó anteriormente, el estudio puede basarse también en las oportunidades que se presenten para mejorar continuamente y no solo en el indicador económico ya que existen casos en que el valor de la inversión es retribuido proporcionalmente a la ganancia que se pueda generar así como del tipo de proyecto que se esté manejando.

\section{CONCLUSIONES}

El Puerto de Barranquilla tiene una ubicación estratégica en comparación con los demás puertos nacionales ya que cuenta con el Río y con el Mar Caribe y con base en esto hay que buscar oportunidades de desarrollo y de máximo aprovechamiento ya que hay facilidad para el transporte marítimo y fluvial de mercancía con el país y fuera de este.

No obstante, hay una dificultad en el puerto y es la profundidad del río, ya que trae consigo mucha sedimentación, lo que no permite el paso de buques de manera constante y fluida; desperdiciando así los tratados económicos que existen y sobre todo la ubicación privilegiad mencionada. Esto es algo sobre lo cual se está trabajando constantemente ya que una mejor inversión y un cambio de infraestructura pueden generar mayores ingresos a la ciudad y reconocimiento a nivel nacional e internacional generando competitividad comercial y siendo coherentes con el auge tecnológico y el crecimiento que se viene dando en todas las sociedad económicas del mundo.

Dado lo anterior, se debe generar soluciones ya que el puerto puede tener la capacidad de transportar todo tipo de embarcación, por lo que dado el estudio de caso se decidió tomar la opción 1 de la Alternativa 1 que es realizar obras en Isla 1972 y Bocas de ceniza con espolones en pilotes de acero y núcleos en Bolsacretos, cerrando el dique en arena y haciendo espolones en roca. Con este se generaría mayor profundidad del puerto, de 28 pies pasaría a 36 pies, permitiendo el paso de embarcaciones con mayor calado y dado el nuevo puente con mayor altura podría darse mayor uso de la extensión del río Magdalena generando mayor interconectividad entre puertos de la sociedad regional y los demás puertos de otras ciudades. No obstante, y como se mencionó anteriormente en este artículo, hay decisiones que se pueden tomar no solo de los valores que genera un análisis financiero o matemático, ya que el dinero tiene valor en el tiempo y es bueno observar si hay factores que permitan hacer un análisis diferente como es el de observar el costo de oportunidad que genera una opción. Es por eso que no se cierra la posibilidad de tomar la fase 2 de la Alternativa 2 que genera una apertura 
de mercado. Una menor inversión podría generar menores ingresos, dado esto, si se mejora la infraestructura de manera total pueden obtenerse buenos resultados. En conclusión, siempre se debe mirar en pro del mejoramiento continuo, de la superación de crisis y darle paso a la toma de decisiones rentables para el proyecto que se esté estudiando. Aquí, se busca la mejor alternativa para el puerto de Barranquilla para permitir un mayor transporte de mercancías, posicionar al puerto y facilitar el comercio nacional e internacional.

\section{REFERENCIAS}

[1] S. Díaz Lucero , S. Guerrero Pérez, Y. Vega Sampayo y E. Olivero Vega, «Estrategias para mejorar la infraestructura del puerto de Barranquilla.,» Liderazgo Estratégico, vol. 8, nº 1, pp. 78-93, 2018.

[2] A. Otero, «El puerto de Barranquilla: retos y recomendaciones.,» Revista de Economía del Caribe, $\mathrm{n}^{\circ}$ 10, pp. 126$159,2012$.

[3] G. Piraquive Galeano, M. Matamoros Cárdenas, E. Cespedes Rangel y J. Rodriguez Chacon, «Archivos de Economía: Actualización de la tasa de rendimiento del capital en Colombia bajo la metodología de Harberger.,» 008 Agosto 2018. [En línea]. Available: https://colaboracion.dnp.gov.co. [Último acceso: 24 Noviembre 2019].

[4] M. Otero De la Vega y N. Mauricio Ramírez, Trabajo de grado: Puerto de Barranquilla, Bogotá, 2018.

[5] M.Nuñez, J.Correa, G.Herrera, P.Gómez, S.Morón \& N. Fonseca, "Estudio de percepción sobre energía limpia y auto sostenible," IJMSOR, vol. 3, no. 1, pp. 11-15, 2018. Recuperado de http://ijmsoridi.com/index.php/ijmsor/article/view/89 